\title{
Pathophysiology of peripheral nerve injury: a brief review
}

\author{
Mark G. BurnetT, M.D., AND Eric L. Zager, M.D. \\ Department of Neurosurgery, Hospital of the University of Pennsylvania, Philadelphia, Pennsylvania
}

\begin{abstract}
Clinicians caring for patients with brachial plexus and other nerve injuries must possess a clear understanding of the peripheral nervous system's response to trauma. In this article, the authors briefly review peripheral nerve injury (PNI) types, discuss the common injury classification schemes, and describe the dynamic processes of degeneration and reinnervation that characterize the PNI response.
\end{abstract}

\section{KEY WORDS • peripheral nerve • nerve injury • neurapraxia • axonotmesis • neurotmesis}

After a nerve is injured in the periphery, a complex and finely regulated sequence of events commences to remove the damaged tissue and begin the reparative process. Unlike cellular repair in other areas of the body, the response of the peripheral nerve to injury does not involve mitosis and cellular proliferation. Our understanding of the peripheral nerve regeneration process has increased significantly during the past several decades concurrent with advances in cellular and molecular biology. We now see that a nerve's response to injury is not simply localized at the site of damage but involves the cellular bodies located in the spinal cord and ganglia as well. Critical roles are played by Schwann cells as well as macrophages and inflammatory cells. The importance of neurotrophic factors has also been elucidated. We provide a review of PNI and repair processes and illustrate the remarkable cooperation between destructive and restorative forces that must occur if proper nerve regeneration is to take place.

\section{BASIC INJURY TYPES}

Before discussing the pathophysiology of PNIs, it is important to provide a brief description of the basic injury types seen in clinical practice.

Stretch-related injuries are the most common type. Peripheral nerves are inherently elastic because of their collagenous endoneurium, but when traction forces exceed the nerve's capacity to stretch, injury occurs. ${ }^{17}$ If the force applied is great enough, a complete loss of continuity may occur as in a brachial plexus avulsion. More often, however, continuity is retained. Injuries of this type can be seen in isolation (for example, in Erb palsy and similar brachial plexus birth-related injuries) or in association with extremity fractures at points where nerves and bone are in close approximation (for example, in radial nerve injury following humeral fracture.

Abbreviations used in this paper: $\mathrm{CNS}=$ central nervous system; $\mathrm{NGF}=$ nerve growth factor; $\mathrm{PNI}=$ peripheral nerve injury.
Lacerations such as those created by a knife blade are another common PNI type, comprising 30\% of serious injuries in some series. ${ }^{5}$ Whereas these can be complete transections, more often some nerve element of continuity remains. Because most research models involve a laceration-type injury mechanism because it is easily reproduced, the details of nerve degeneration and regeneration discussed in this article are perhaps most representative of this injury type.

Compression is a third common type of PNI. These injuries include the "Saturday Night palsy" due to radial nerve compression as well as entrapment neuropathies and do not involve a severance or tearing of the neural elements. Total loss of both motor and sensory function may occur, but the pathophysiology responsible for these deficits is unclear because complete nerve continuity is maintained. Two pathological mechanisms are believed to be involved in these injuries: mechanical compression and ischemia. Discerning which mechanism is more important in compression injuries has been difficult. The authors of tourniquet experiments performed in the 1930s demonstrated that, at least in short-term compression, ischemia and not the pressure itself causes the resultant physiological conduction block. ${ }^{8}$ Exactly how short-term ischemia induces such a block has not been determined, but large myelinated fibers appear to be more susceptible to ischemic effects than smaller unmyelinated ones. Little or no histological change is seen as a result of such injuries, and the effects appear to be reversible unless ischemia persists for more than approximately 8 hours.

Mechanical deformation is thought to be the primary mechanism in the more severe cases of compression injury such as in Saturday Night palsy in which function may be lost for weeks and full recovery does not always occur. Experiments involving a pneumatic cuff to recreate this sort of compression injury have and shown that nerves undergo degenerative changes at the edges of the compressed area and not under the center of the cuff where ischemia is most severe. ${ }^{10}$ Ultrastructural examination of 
the nerves has demonstrated that the axoplasm and myelin beneath the cuff were pushed away from the point of greatest compression and toward the edges of the cuff, reinforcing the finding that mechanical deformation is responsible in these injuries. ${ }^{11}$

\section{NERVE INJURY CLASSIFICATION}

The timing and success of the peripheral nerve repair process depends on the extent of injury. Clinically useful injury grading systems have been developed that allow correlation of the microscopic changes occurring after nerve injury and patient symptomatology. Perhaps the most widely accepted are those developed by Seddon ${ }^{15}$ and Sunderland ${ }^{18}$ (Fig. 1). Seddon divided nerve injuries by severity into three broad categories: neurapraxia, axonotmesis, and neurotmesis. Neurapraxia, the mildest injury type, does not involve loss of nerve continuity and causes functional loss, which is transient. This symptom's transience it thought to be due to a local ion-induced conduction block at the injury site, although subtle alterations in myelin structure have also been found. Axonotmesis occurs when there is complete interruption of the nerve axon and surrounding myelin while the surrounding mesenchymal structures including the perineurium and epineurium, are preserved. Axon and myelin degeneration occur distal to the point of injury, causing complete denervation. The prospect of recovery is excellent in such injuries because of the remaining uninjured mesenchymal latticework that provides a path for subsequent sprouting axons to reinnervate their target organ. Neurotmesis involves disconnection of a nerve. Functional loss is complete and recovery without surgical intervention, does not usually occur because of scar formation and the loss of the mesenchymal guide that properly directs axonal regrowth.

Sunderland's classification system ${ }^{18}$ further stratifies the three injury types described by Seddon ${ }^{15}$ into five categories according to severity. A first-degree injury is equivalent to Seddon's neurapraxia and a second-degree injury is equivalent to axonotmesis. Third-degree nerve injuries occur when there is disruption of the axon (axon-

\section{Peripheral Nerve Injury Classification}

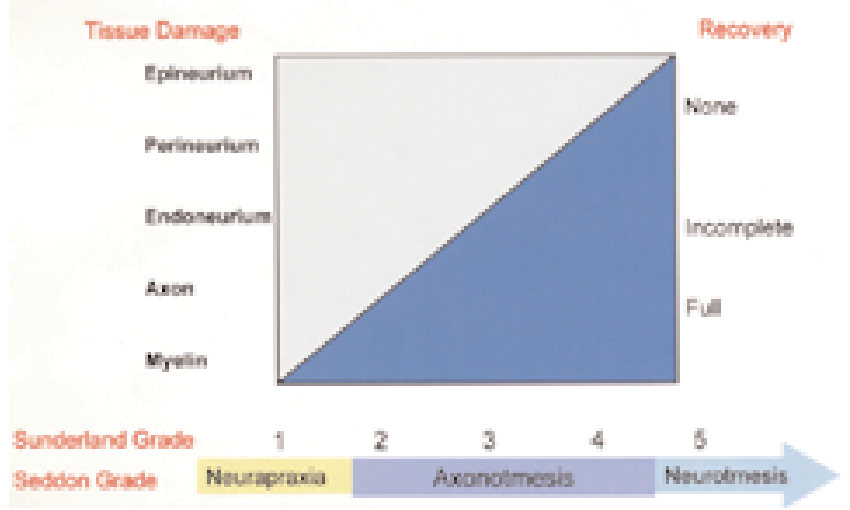

Fig. 1. Graph illustrating the Sunderland and Seddon PNI grading systems. Gradations in both systems are associated with the anatomical extent of injury and with the chance of a spontaneous recovery of function after trauma. otmesis) and also partial injury to the endoneurium. This categorization places a third-degree between Seddon's axonotmesis and neurotmesis. Dependent on the extent of the endoneurial damage, functional recovery may be possible. Sunderland divides Seddon's neurotmesis into fourth- and fifth-degree injuries. In a fourth-degree injury, all portions of the nerve are disrupted except the epineurium. Recovery is not possible without surgical intervention. Similarly, a fifth-degree injury involves complete severance of the nerve.

\section{NEURAL RESPONSE TO INJURY}

Before regeneration of nerve fibers can occur, a series of degenerative processes must take place, many of which are direct preludes to regeneration. The success of regeneration depends largely on the severity of the initial injury and resultant degenerative changes. Pathological changes are mild or absent in first-degree injuries in which the mechanism is conduction block alone, and no true degeneration or regeneration occurs. In second-degree injuries (axonotmesis) there is little histological change at the injury site or proximal to it; however, distal to the injury site, a calcium-mediated process known as Wallerian (or anterograde) degeneration is known to occur ${ }^{22}$ (Fig. 2).

In Wallerian degeneration the primary histological change involves physical fragmentation of both axons and
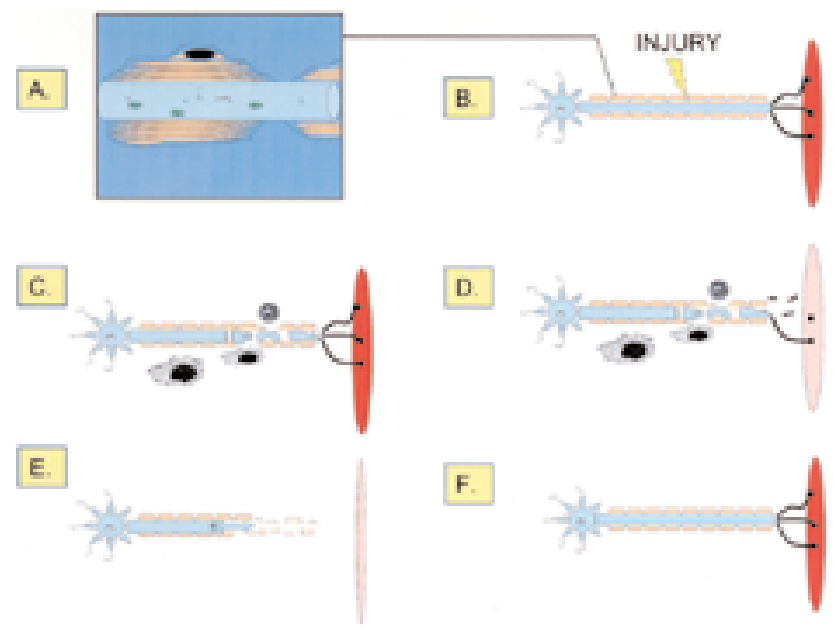

Fig. 2. Illustrations showing the Wallerian degeneration of the peripheral nerve. In the normal (A) and injury state (B), Schwann cells align along the length of the axon forming multiple layers of myelin. The characteristic spaces between neighboring Schwann cells are known as nodes of Ranvier. After significant injury (C), the nerve will begin to degrade in an anterograde fashion. The axon and surrounding myelin break down during this process. The round mast cells can be seen as can the phagocytic macrophages that interact with Schwann cells to remove the injured tissue debris. As the degradation of the distal nerve segment continues (D), connection with the target muscle is lost, leading to muscle atrophy and fibrosis. Once the degenerative events are complete (E), all that remains is a column of collapsed Schwann cells (bands of Büngner). Axon sprouts with a fingerlike growth cone advance using the Schwann cells as guides. After reinnervation (F), the newly connected axon matures and the preinjury cytoarchitecture and function are restored. 
myelin, a process that begins within hours of injury. Ultrastructurally, both neurotubules and neurofilaments become disarrayed, and axonal contour becomes irregular, due to varicose swellings. By 48 to 96 hours postinjury, axonal continuity is lost and conduction of impulses is no longer possible. Myelin disintegration lags slightly behind that of axons but is well advanced by 36 to 48 hours.

Schwann cells play a key role in Wallerian degeneration. They initially become active within 24 hours of injury, exhibiting nuclear and cytoplasmic enlargement as well as an increased mitotic rate. These cells divide rapidly to form dedifferentiated daughter cells that upregulate gene expression for a multitude of molecules to assist in the degeneration and repair process. An initial Schwann cell role is to help remove the degenerated axonal and myelin debris and then pass it on to macrophages. The macrophages have migrated into the traumatized region, primarily through a hemopoietic route, passing through the walls of capillaries, which have become permeable in the injury zone. Schwann cells and macrophages work together to phagocytose and clear the site of injury in a process that requires 1 week to several months.

Endoneurial mast cells also play a pivotal role in this process, proliferating markedly within the first 2 weeks postinjury. They release histamine and serotonin, which enhance capillary permeability and facilitate macrophage migration. During the initial stages, the endoneurial tubes swell in response to the trauma, but after the first 2 weeks they become smaller in diameter. By 5 to 8 weeks, the degenerative process is usually complete, and nerve fiber remnants composed of Schwann cells within an endoneurial sheath are all that remain.

In third-degree injuries, a more significant trauma-induced local reaction occurs. These intrafascicular injuries involve retraction of the severed nerve fiber ends due to the elastic endoneurium. Local vascular trauma leads to hemorrhage and edema, which result in a vigorous inflammatory response. Fibroblasts proliferate, and a dense fibrous scar causes a fusiform swelling of the injured segment. Interfascicular scar tissue also develops so that the entire nerve trunk, which is left in continuity, is permanently enlarged. Often, it is adherent to perineural scar tissue as well.

\section{Distal Segment}

Distal to the injured segment, Wallerian degeneration follows a sequence very similar to that observed in second-degree injuries. One important difference is that the intrafascicular injury impairs axonal regeneration and, therefore, the endoneurial tubes remain denervated for prolonged periods. While denervated, the endoneurial tubes begin to shrink in a process that reaches a maximum at approximately 3 to 4 months postinjury. ${ }^{17,19}$ The endoneurial sheath progressively thickens secondary to collagen deposition along the outer surface of the Schwann cell basement membrane. If the endoneurial tube does not receive a regenerating axon, progressive fibrosis ultimately obliterates it.

Stacks of Schwann cell processes representing collapsed endoneurial tubes become microscopically visible late in the Wallerian degeneration progress in these more significant injuries. The Schwann cell columns are known as the bands of Büngner and become important guides for sprouting axons during reinnervation. These bands provide an early illustration of a second role for Schwann cells after nerve injury, the neurosupportive role for axon regrowth. This will be described further when nerve regeneration is discussed.

In fourth- and fifth-degree injuries, local reaction to the severe trauma is pronounced. Endoneurial tubes, as well as fasciculi, are disrupted and Schwann cells and axons are no longer confined. The epineurium is also damaged and reactive epineurial fibroblasts are present at the severed ends within 24 hours. These are accompanied by proliferating Schwann cells and peri- and endoneurial fibroblasts. Vigorous cellular proliferation peaks within the 1 st week and continues for a prolonged period. As in the aforementioned milder injuries, capillary permeability increases, probably as a result of mast cell degranulation, and edema and macrophage infiltration follow. The magnitude of this response correlates with the severity of the trauma to both the nerve and the surrounding tissues.

In fourth- and fifth-degree injuries, the nerve ends become a swollen mass of disorganized Schwann cells, capillaries, fibroblasts, macrophages, and collagen fibers. Regenerating axons reach the swollen bulb of the proximal stump and encounter formidable barriers to further growth. Many axons form whorls within the scar tissue or are turned back along the proximal segment or out into the surrounding tissue. Some of the regenerating axons may reach the distal stump, an accomplishment that is dependent on multiple factors, including the severity of the original injury, the extent of the scar formation, and the delay before the axons reach the injury site. As in third-degree injuries, endoneurial tubes left unoccupied for prolonged periods undergo progressive shrinkage and fibrosis, ultimately becoming completely obliterated by collagen fibers.

\section{Proximal Segment and Cell Body}

Changes in neuronal cell bodies and in nerve fibers proximal to the site of injury depend on the severity of the injury as well as the proximity of the injured segment to the cell body. Schwann cells inevitably degrade along the proximal segment near the area of injury, and axons and myelin become visibly reduced in diameter. This proximal degradation can be minimal (ranging from the injury site back to the next node of Ranvier) or it can extend all the way back to the cellular body. If the cellular body actually degenerates, which may occur in severe trauma, the entire proximal segment undergoes Wallerian degeneration and is phagocytosed.

Following significant injury, the proximal segment axon is reduced in diameter, particularly if functional connections to appropriate end organs are not reestablished. Nerve conduction velocity is accordingly reduced. As regeneration proceeds, the axonal diameter increases, but may never reach normal preinjury levels. A definite interdependence exists between the cellular body and the axon in terms of recovery: the cellular body does not recover fully without the reestablishment of functional peripheral connections, and the final axonal caliber depends to a great extent on the recovery of the cellular body.

The nerve cell body itself reacts to axonal injury in a 
relatively predictable fashion. Within 6 hours of the injury, the nucleus migrates to the periphery of the cell and Nissl granules, rough endoplasmic reticulum, break up and disperse. This process is called chromatolysis. Simultaneously, there is a brisk proliferative response of perineuronal glial cells, most likely signaled in some manner by the process of chromatolysis. Glial cell processes extend to the affected neuron and interrupt synaptic connections, possibly to isolate the neuron for its recovery phase.

Cell survival is not assured after severe nerve injury. The incidence of apoptosis-related cell death in dorsal root ganglion neurons following axonotmesis ranges from 20 to $50 \% .{ }^{9}$ Death occurs more frequently if axonotmesis occurs proximally and in injuries involving sensory or cranial nerves. This process of injury-induced neuronal cell death remains poorly understood, but conditions within the microenvironment of the injury site are believed to be important. In fact, the regenerative capacity of the peripheral nerve's microenvironment has been studied since the early 1900s when Cajal proposed that it was the peripheral nerve milieu rather than intrinsic neuronal differences been peripheral and central nervous system neurons that explained the failure of regeneration in the latter. Since that time researchers have demonstrated that central neurons have the capacity to regenerate in a peripheral environment and that peripheral neurons lose this ability when placed in a central environment. ${ }^{2,13}$ The authors of molecular studies continue to elucidate the supportive role of Schwann cells and have demonstrated the presence of trophic molecules in the peripheral nerve microenvironment such as NGF, brain-derived neurotrophic factor, and others that appear to influence cell survival after injury. ${ }^{21,23,24}$

\section{NERVE REGENERATION}

In severe injuries nerve regeneration begins only after Wallerian degeneration has run its course, but in mild injuries the regenerative and repair processes begin almost immediately. For first- and second-degree injuries (neurapraxia and axonotmesis), restoration of function is the rule. This occurs early via reversal of conduction block or late via axonal regeneration. Functional recovery is complete in these milder degrees of injury. Both morphological and physiological changes are fully reversible.

In more severe nerve injuries in which endoneurial tubes are disrupted, regenerating axons are no longer confined to their original sheaths. They may meander into surrounding tissue or into inappropriate endoneurial tubes, thus failing to reinnervate their proper end organs. Neurological recovery is compromised, generally to a degree proportional to the severity of the injury.

Functional recovery after nerve injury involves a complex series of steps, each of which may delay or impair the regenerative process. In cases involving any degree of nerve injury, it is useful initially to categorize these regenerative steps anatomically on a gross level. The sequence of regeneration may be divided into anatomical zones: 1 ) the neuronal cell body; 2) the segment between the cell body and the injury site; 3) the injury site itself; 4) the distal segment between the injury site and the end organ; and 5) the end organ itself. A delay in regeneration or unsuccessful regeneration may be attributed to pathological changes that impede normal reparative processes at one or more of these zones.

The regeneration and repair phase following nerve injury may last for many months. The earliest signs of this phase are visible changes in the cell body that mark the reversal of chromatolysis. The nucleus returns to the cell center and nucleoproteins reorganize into the compact Nissl granules. Postinjury, many subcellular metabolic functions were altered during chromatolysis. Likewise, RNA synthesis was increased and neurotransmitter synthesis decreased. Chromatolysis heralded a fundamental shift in cell function from synaptic transmission to cellular repair. The metabolic machinery was reprogrammed so that the cell would be able to produce the vast amount of protein and lipid needed for axonal regrowth during the regeneration phase.

A complex and incompletely understood interaction occurs between the cell body and the regenerating axon tip. Axoplasm, which serves to regenerate the axon tip, arises from the proximal axon segment and cell body. Both fast and slow components of axoplasmic transport supply materials from the cell body to the sites of axonal regeneration. The rate of increase in protein and lipid synthesis in the cell body influences the rate of advance and the final caliber of the regenerating axon. The human peripheral neuron's capacity to initiate a regenerative response appears to persist for at least 12 months after injury, and a robust response can be elicited even after repeated injuries.

The length of the segment between the regenerating axon tip and the injury site depends on the severity of the original injury and the consequent retrograde degradation. The first signs of axon regrowth in this segment may be seen as early as 24 hours postinjury, or they may be delayed for weeks in more severe injury. The rate of axonal regrowth is determined by changes within the cell body, the activity of the specialized growth cone at the tip of each axon sprout, and the resistance of the injured tissue between cell body and end organ.

There may be multiple axon sprouts within each endoneurial sheath, even in milder injuries, that do not involve destruction of the sheath itself. The fate of these multiple sprouts is not clear even in experimental paradigms. The timing of degenerative and regenerative processes is such that there must be a significant overlap between these in certain segments. For example, in milder injuries in which there is no significant delay in regeneration across the injury site, the growth cone at the advancing axon tip must encounter the debris of Wallerian degeneration in the distal segment. This debris does not appear to impede regeneration, perhaps because the growth cone secretes a protease that can help dissolve material blocking its path. ${ }^{6}$

In very proximal injuries in which there is considerable delay before the advancing axon tip reaches the distal segment, the empty endoneurial tubes distally have decreased in diameter. This factor may be responsible, in part, for a terminal slowing in axonal regrowth. Surgical intervention that interrupts entering nutrient arteries does not appear to impair axonal regeneration, provided that longitudinal arteries within the nerve itself are not disrupted.

In severe nerve injuries that disrupt the endoneurial 
tubes, nerve fascicles, or trunks, formidable obstacles face the regenerating axons that reach the injury site. There may be a gap between the disrupted nerve ends, allowing regenerating axon sprouts to wander into surrounding tissue. Scarring is inevitably present at the site of severe injury; the extent depends on multiple factors including the timing of the arrival of the regenerating sprouts after injury.

It has been well documented that regenerating axons may at times successfully traverse long gaps spontaneously, despite the presence of substantial scar tissue; however, there is no question that appropriate surgical repair can eliminate the gap and reduce the amount of intervening scar tissue. This procedure provides no guarantee of proper fascicle orientation, of course, and regenerating axons may grow into functionally inappropriate endoneurial tubes or even may fail to reenter an endoneurial tube. Either circumstance results in wasted axons.

Previously nonmyelinated axons may regenerate into endoneurial sheaths that formerly contained myelinated axons (and vice versa). This regeneration will not be wasteful. The resistance that an axon meets at the injury site results in the formation of multiple smaller axon sprouts. These daughter axons do not all make their way into the distal segment. No specific neurotropism is known to enhance the growth of a regenerating axon into its original endoneurial tube, but some form of neurotropic influence has been demonstrated in experimental paradigms. Scarring within the bridging tissue impedes the regeneration and misdirects axon sprouts in to functionally unrelated endoneurial tubes. Residual scar tissue also interferes with the maturational processes of axons that do negotiate the injury site.

Axons that successfully enter the endoneurial tubes in the segment distal to the injury site stand a good chance of reaching the end organ, given reasonable growth conditions. The distal regeneration rate is slower if the endoneurial tubes have been disrupted because axon sprouts must first find their way into the tubes before advancing. The specialized growth cone at the tip of each axon sprout contains multiple filopodia that adhere to the basal lamina of the Schwann cell and use it as a guide. Both contact and chemotactic guidance have been shown to be important in directing advancement of the growth cone. . $^{3,4}$ At times, because several small axon sprouts may enter the same endoneurial tube, a regenerated nerve fiber may contain more axons than the original nerve.

If a functionally unrelated end organ is reached, further development of the axon and remyelination do not occur. Similarly, axonal development and maturation are aborted if the end organ, due to prolonged denervation, has undergone degenerative changes that do not allow the establishment of functional connections. If the entry of regenerating axons into the distal segment is delayed more than approximately 4 months, the axons are entering endoneurial tubes of smaller diameter, generally $3 \mu \mathrm{m}$ or less. This shrinkage may make it more difficult for axon sprouts to locate and enter endoneurial tubes, but this does not appear to impede axonal regrowth once sprouts are inside the tubes. This is presumably due to the elastic properties of the endoneurium.

The return of function does not require absolutely faith- ful recovery of nerve architecture. The effects of prolonged denervation, which do appear to impair functional recovery, are at the level of the injury site-that is, preventing the regenerating axons from entering appropriate endoneurial tubes-or at the end organ.

End organ undergoes characteristic histological changes with nerve degeneration and subsequent reinnervation. Muscle fibers atrophy quite rapidly (a mean $70 \%$ reduction of cross-sectional area by 2 months) and cell nuclei assume a central rather than the normal peripheral position. The synaptic folds of motor endplates are preserved for at least 1 year after denervation.

Tremendous proliferation of fibroblasts also characterizes the histological picture of denervation. New collagen is deposited in both the endo- and perimysium. In general, muscle fibers are not replaced by connective tissue but rather atrophied fibers are separated by thickened connective tissue, so that the overall internal pattern of muscle architecture is preserved. Occasional dropout of muscle fibers does occur. This is a relatively late phenomenon, generally observed between 6 and 12 months after denervation.

Regenerating axonal sprouts follow the original Schwann cells to the denervated motor endplates to reform neuromuscular junctions. ${ }^{14}$ Collateral sprouting also occurs, resulting in groups of reinnervated muscle fibers, all of the same fast or slow types. This is a characteristic finding in reinnervated muscle, contrasting sharply with the random pattern observed in normal muscle.

Unfortunately, incomplete motor recovery occurs commonly after moderate-to-severe nerve injuries. This is due to a number of factors within the muscle itself and in the regenerating nerve. Intramuscular fibrosis may limit the efficacy of the contraction produced by a nerve impulse. Appropriate physical therapy can help maintain the denervated muscles in an optimal condition to receive the regenerating axon terminals.

Functional motor recovery is obviously impaired if significant numbers of axons do not successfully reform functional connections with the muscle. Even if the numbers are adequate, erroneous cross-reinnervation may produce a suboptimal functional result: an originally "fast" muscle may be reinnervated by axons previously innervating "slow" muscle, and the result may be a mixed form with inefficient contraction.

In cases in which significant motor recovery occurs, functional outcome may be impaired by concomitant sensory deficits, particularly in proprioception. Denervated sensory receptors survive and may make useful functional recoveries after 1 year and possibly after many years. In first- and second-degree injuries, return of sensation is complete in its original pattern, even after 6 to 12 months of denervation. This is due to faithful reinnervation of sensory receptors by their original axons.

After more severe injuries and nerve repair, sensory recovery is never complete. This is undoubtedly related to a combination of factors, including failure of sensory axons to reach the skin, cross-reinnervation (an axon originally from one type of receptor making connections with a different type of receptor), and possibly degeneration of sensory receptors. Sensory reinnervation appears to be modality specific, but it is less so than motor reinnervation, 
which means that sensory cross-reinnervation is unfortunately more common. Some controversy exists over the fate of denervated encapsulated sensory receptors. These receptors include Pacinian corpuscles and Meissner corpuscles, which are rapidly adapting receptors mediating light touch and vibration, as well as Merkel cells, which are slowly adapting receptors that mediate constant touch and pressure. It is believed that these specialized receptors survive in an atrophied state for prolonged periods, awaiting the arrival of an appropriate nerve terminal. The survival period has not been clearly established, however, and there is some evidence indicating that the protective sensation, which recovers years after denervation, is mediated by less elaborate sensory receptors.

The rate of axonal regeneration has been assumed to be constant and, in clinical situations, is generally estimated to be $1 \mathrm{~mm}$ per day and is often followed by an advancing Tinel sign. Reported rates of regeneration, however, vary broadly from 0.5 to $9 \mathrm{~mm}$ per day. This variability is due to several factors. 1) The rate of axon growth decreases with increasing distance from the cell body to the advancing axon tip. 2) Measurements of axonal regeneration were made in different species after different methods of nerve injury. 3) The techniques for measuring regeneration were different (for example, Tinel sign compared with functional recovery). Moreover, the rate of regeneration can depend on the nature and severity of the nerve injury, the duration of denervation, and the condition of the peripheral tissues. Regeneration after surgical nerve repair is slower than uncomplicated regeneration, most likely reflecting the severity of the original injury. Aging has also been shown to retard the rate of axonal regrowth.

Axonal regeneration is not synonymous with return of function. A process of maturation precedes functional recovery. Morphological changes of maturation proceed along the regenerating axon at a slower rate than axon regrowth and continue for a protracted period - as long as 1 year. Remyelination develops in a manner similar to that for developing nerve fibers, involving alignment of Schwann cells and encircling of the axon to form a multilamellated sheath. This process begins within 2 weeks of the onset of axonal regeneration and results in myelinated axons quite similar to the originals except with shortened internodes. The axon's diameter increases progressively until normal dimensions are reached, but this enlargement is dependent on the establishment of functional connections between the axon tip and the appropriate end organ.

\section{NEUROTROPHIC FACTORS}

Ensuring that the processes of degeneration and regeneration proceed in an orderly fashion requires a sophisticated system of cellular communication. Researchers have long suspected the existence of a cascade of cell-signaling molecules and trophic factors similar to those seen in an inflammatory response. Neurotrophic factors such as NGF, brain-derived neurotrophic factor, ciliary neurotrophic factor, and many others have been identified and are thought to be important in the nerve repair process. ${ }^{1,14,23,24}$

Nerve growth factor was the first neurotrophic molecule to be identified and remains the best characterized. ${ }^{7}$ It is involved in nerve cell survival and maintenance in the normal state and appears to be an important component of the nerve repair process as well. Factors such as NGF are tonically released from peripheral nerve target organs and transferred to the nerve cell body via retrograde axonal transport. ${ }^{16}$ It may be that the decrease in NGF and other trophic factors reaching the cell body as a result of axonal disruption occurring immediately after nerve injury is the molecular signal needed to trigger the repair process. In one experiment NGF was applied to neurons following axotomy, and this appeared to reverse the cell body changes seen in chromatolysis. ${ }^{12}$ Soon after injury, the amounts of NGF and NGF messenger RNA are greatly increased, consistent with their role as a neurotroph. It is thought that invading macrophages stimulate NGF production via interleukin-1 $\beta$ release, which suggests that the macrophage is involved with both phagocytosis and regeneration. Similarly, Schwann cells have been shown to produce neurotrophic factors, including NGF, at the site of injury.

Neurotrophic factors, like other cell-signaling molecules, bind to specific tyrosine kinase receptors transmitting a signal which ultimately regulates gene activation. Nerve growth factor receptor concentration on the Schwann cells forming the bands of Büngner increases after injury. The NGF that binds to these receptors on the Schwann cells is presented to the regrowing axon sprouts. This NGF uptaken by the axon is then transferred retrograde from the growth cone to the cell body, providing a continued stimulus for growth as well as a guide for the advancing axon. ${ }^{20}$

\section{CONCLUSIONS}

The peripheral nerve response to injury is unique. Neurons of the CNS do not show the same ability to regenerate lost connections. Rather than rebuild, neurons of the CNS circumvent damage by strengthening and reprogramming uninjured pathways so that they conduct the functions that have been lost. The complicated processes of peripheral nerve degeneration and regeneration that have been discussed in this paper are only partly understood. Further investigations into this area will undoubtedly yield information that will lead to surgical and therapeutic advances for the treatment of PNI such as the use of neurotrophic factors to stimulate growth and direct growing axons to their proper target organ. Perhaps this additional understanding will also help explain why it is that the CNS has not developed such a restorative process and illuminate some possible strategies for simulating the peripheral nerve regenerative microenvironment in the brain and spinal cord.

\section{References}

1. Barbin G, Manthorpe M, Varon S: Purification of the chick eye ciliary neuronotrophic factor. J Neurochem 43:1468-1478, 1984

2. David S, Aguayo AJ: Axonal elongation into peripheral nervous system "bridges" after central nervous system injury in adult rats. Science 214:931-933, 1981

3. Dodd J, Jessell TM: Axon guidance and the patterning of neuronal projections in vertebrates. Science 242:692-699, 1988 


\section{Pathophysiology of peripheral nerve injury: a brief review}

4. Gundersen RW, Barrett JN: Characterization of the turning response of dorsal root neurites toward nerve growth factor. $\mathbf{J}$ Cell Biol 87:546-554, 1980

5. Jacques L, Kline DG: Response of the peripheral nerve to physical injury, Crockard A, Hayward R, Hoff JT (eds): Neurosurgery: The Scientific Basis of Clinical Practice, ed 3. London: Blackwell, 2000, Vol 1, pp 516-525

6. Krystosek A, Seeds NW: Plasminogen activator release at the neuronal growth cone. Science 213:1532-1534, 1981

7. Levi-Montalcini R, Hamburger V: Selective growth stimulating effects of mouse sarcoma on the sensory and sympathetic nervous system of the chick embryo. J Exp Zool 116:321-361, 1951

8. Lewis T, Pickering GW, Rothschild P: Centripetal paralysis arising out of arrested bloodflow to the limb. Heart 16:1-32, 1931

9. Lundborg G: A 25-year perspective of peripheral nerve surgery: evolving neuroscientific concepts and clinical significance. J Hand Surg Am 25:391-414, 2000

10. Ochoa J, Danta G, Fowler TJ, et al: Nature of the nerve lesion caused by a pneumatic tourniquet. Nature 233:265-266, 1971

11. Ochoa J, Fowler TJ, Gilliatt RW: Anatomical changes in peripheral nerves compressed by a pneumatic tourniquet. J Anat 113:433-455, 1972

12. Otto D, Unsicker K, Grothe C: Pharmacological effects of nerve growth factor and fibroblast growth factor applied to the transectioned sciatic nerve on neuron death in adult rat dorsal root ganglia. Neurosci Lett 83:156-160, 1987

13. Richardson PM, McGuinness UM, Aguayo AJ: Axons from CNS neurons regenerate into PNS grafts. Nature 284:264-265, 1980

14. Seckel BR: Enhancement of peripheral nerve regeneration. Muscle Nerve 13:785-800, 1990

15. Seddon HJ: Three types of nerve injury. Brain 66:237-288, 1943
16. Slack JR, Hopkins WG, Pockett S: Evidence for a motor nerve growth factor. Muscle Nerve 6:243-252, 1983

17. Sunderland S: The anatomy and physiology of nerve injury. Muscle Nerve 13:771-784, 1990

18. Sunderland S: Nerves and Nerve Injuries, ed 2. London: Churchill Livingston, 1978

19. Sunderland S, Bradley KC: Endoneurial tube shrinkage in the distal segment of a severed nerve. J Comp Neurol 93: 411-420, 1950

20. Taniuchi M, Clark HB, Schweitzer JB, et al: Expression of nerve growth factor receptors by Schwann cells of axotomized peripheral nerves: ultrastructural location, suppression by axonal contact, and binding properties. J Neurosci 8:664-681, 1988

21. Varon SS, Bunge RP: Trophic mechanisms in the peripheral nervous system. Annu Rev Neurosci 1:327-361, 1978

22. Waller A: Experiments on the section of the glossopharyngeal and hypoglossal nerves of the frog, and observations of the alterations produced thereby in the structure of their primitive fibers. Phil Trans Roy Soc 140:423-429, 1850

23. Yan Q, Elliott J, Snider WD: Brain-derived neurotrophic factor rescues spinal motor neurons from axotomy-induced cell death. Nature 360:753-755, 1992

24. Yin Q, Kemp GJ, Frostick SP: Neurotrophins, neurones and peripheral nerve regeneration. J Hand Surg Br 23:433-437, 1998

Manuscript received March 4, 2004.

Accepted in final form April 2, 2004.

Address reprint requests to: Mark G. Burnett, M.D., Department of Neurosurgery, Hospital of the University of Pennsylvania, Silverstein Building 5th Floor, 3400 Spruce Street, Philadelphia, Pennsylvania 19104. email: burnettmg@ hotmail.com. 